ingredients in closer and longer contact than water. As to the glands and laminæ of the urethra, it is probable that the thick fluid matter enters them more easily after they have been dilated by the bougie. Of the bougies described, I use Nos. 18 to 23 , French (Charrière). I never use smaller than No. 18 , but prepare the urethra with soft bougies until this size will pass. As soon as this is accomplished I begin the cure with my grooved bougies, which I pass daily. If there is difficulty in passing No. 18, I employ the same sized bougie the next day, and then pass on to No. 19, \&c.

After using these bougies I have never witnessed a case of chill or fever, but I will not say that this could not occur just as after passing ordinary bougies. The pain or disagreeable feeling is not greater than that caused by the introduction of any other instrument into the urethra. Nay, the well-known burning in the urethra frequently experienced by patients in passing urine after the first application of the bougie will often be entirely absent. The longer the bougie is left in, the more favourable is the effect on the infiltration. Some patients Ieel burning and tenesmus after a few minutes, others after half an hour, and others still later. The time during which the bougie may remain in is dependent on these circumstances. Although the salve is so prepared that it cannot injure the normal parts, I avoid introducing it unnecessarily into the posterior portion of the urethra, whence it would flow into the bladder. I effect this by passing the bougie, in a case of gonorrhoea anterior, only so far that its point just touches the musculus sphincter vesice internus. As the grooves with the salve begin at a distance of five centimetres from the point, none or very little of the salve will flow into the posterior urethra. In a case of gonorrhoea posterior I push the bougie further, so that its grooved part lies in the partes membranacea and prostatica. Here we cannot avoid a little of the salve flowing into the bladder. The results I have obtained with this treatment are satisfactory. On an average I succeeded by ten applications to effect a cure of cases of chronic gonorrhcea which had before proved very obstinate. In a few cases twenty and more applications were necessary. Frequently after using a very small number of bougies a striking improvement was visible.

Whether a chronic gonorrhoea which no longer shows a secretion at the meatus will improve can be decided by simply examining the urine with the naked eye. The quantity of gonorrhoeal flakes, their size, and length will diminish with the gradual process of cure. The microscope teaches us that at the beginning the thick flakes are almost entirely composed of pus-corpuscles, while epithelial cells are but few. With the improvement of the chronic gonorrhœa the quantity of pus-corpuscles diminishes, and that of epithelial cells increases, till at last but few puscorpuscles are to be found here and there embedded in the epithelial cells. Thus we are enabled exactly to observe the different stages of chronic gonorrhœa. More than once I could readily, after two applications, perceive a difference in the composition of the flakes in so far as the proportion of epithelial cells to pus-corpuscles had changed in favour of the former. Altogether by this procedure I have cured about fifty obstinate cases of chronic gonorrhœa, which had resisted all other methods: twenty-seven of these had been cases of a year and a half's standing, twelve of more than a year's standing, and others less. Berlin.

\section{THREE CASES OF}

\section{SLOUGHING ULCER OF THE PENIS.}

BY FREDERICK W. LOWNDES, M.R.C.S. ENG., SURGEON TO THE LIVERPOOL LOCK HOSPITAL.

CAst 1.-James P_-, aged twenty-three, a coach-smith, consulted me on Oct. 22 nd last for phimosis and ulcers. The inguinal glands on the left side were indurated. He first noticed the fact that he was sore a week after sexual intercourse, and he had been suffering for three weeks before he came to $m e$, having been under the care of a chemist. Hearing this, I examined him very carefully, and as the prepuce could not be retracted, I syringed it out repeatedly with warm water. There was no offensive odour nor any evidence of slough. I ordered him to inject black wash under the prepuce several times a day, and to take a pill containing two grains each of grey powder and Dover's powder night and morning. Late in the evening of the 24 th I was sent for to see him at his residence in consequence of hæmorrhage. On arrival I found a most offensive. discharge coming from under the prepuce mixed with blood. I at once slit the latter up, and found that a considerable. portion of the glans had sloughed away. The patient stated that the discharge began to be offensive in the evening of the $22 \mathrm{nd}$, and that the bleeding had been going on all the afternoon. I stopped the pills and gave him a mixture containing carbonate of ammonia and laudanum, to be taken every few hours. There was no further hæemorrhage after the glans was fully exposed, and the parts were dressed with lead and opium lotion. The next day he was much better and the slough had separated. I therefore had the parts well dusted over with iodoform powder, which was repeated at short intervals. The patient made a rapid recovery. There was no induration or any other indication of syphilis, and the parts were perfectly healed in a few weeks.

CAsE 2.-John D-_, aged eighteen, a tramcar guard, was admitted into the Lock Hospital on Nov. 9th with phimosis and ulcers. He stated that he began to feel sore four days after sexual intercourse, and that be had been suffering for one month previous to his admission. There had been some hæmorrhage latterly, and he had received no proper medical treatment. I saw him on his admission, and, finding a most offensive discharge, at once divided the prepuce. Part of the corona glandis had sloughed away with a portion of the furrow between it and the inner prepuce, leaving so deep a cavity that I feared lest the urethra should become involved. Iodoform, in fine powder, was applied; a mixture containing carbonate of ammonio and laudanum was given thrice daily; and the patient was ordered to keep in bed. He progressed most favourably until 3 A.M. on the morning of the 14th, when severe hæmorrhage occurred from the wound. The resident superintendent (Mr. Serjeant) was called up, and, finding him in a very weak state, sent for me. On my arrival I found that the hæmorrhage, which had been evidently venous, had ceased, but the patient was in a very exhausted state. Brandy had been administered, and I ordered this to be repeated at short intervals. I cleared away all the clots, cleansed the wound, and plugged it up with lint saturated in a strong solution of perchloride of iron. There being no further hæmorrhage, in a few days the iodoform powder was resumed, and the patient completely recovered. There were no manifestations of syphilis, and it was evidently a case of chancroids taking on sloughing action from neglect, and exposure of the patient to cold and wet in consequence of his calling.

CASE 3.-Wm, D-, aged thirty-eight, seaman, was admitted into the hospital on Dec. 7th with a sloughing ulcer of the furrow, corona glandis, and part of the glans. It was superficial, was of short duration, and separated in three days, leaving a healthy surface. The treatment was the same as in the previous cases, except that strong opium lotion was used at first, followed by iodoform ointment. There was no hæmorrhage whatever.

Cases of sloughing ulcer are much less frequent than they were twenty years ago ; hence the appearance of these three cases within so short a period seems to me to deserve notice. My connexion with the hospital as surgeon extends to ten years, and the comparative rarity of this form of sore with its much greater frequency twenty-five years ago, when I attended as a student, is very striking. During a period of eighteen months I can remember three cases where the whole of the penis was lost, in addition to many others where there was considerable mutilation. My colleague, Dr. Bernard, who saw all the above cases, informs me that during his period of office, now nearly four years, he has had about four cases, two of which were complicated with hæmorrhage. We both rely upon opium, administered internally, as a most valuable remedy; and we also employ iodoform, partly as a very useful application in promoting the healing process, partly as a most powerful deodoriser to the horrible effluvium which generally arises from sloughing ulcers of any kind, wherever situated. A very convenient mode of application is by keeping it finely powdered in a box with a lid perforated like a pepperbox (though not quite so finely), and with one or two Tonquin beans, which render it more pleasant, but no less effective. 